ORIGINAL ARTICLE

\title{
Cancer incidence and mortality of isocyanate exposed workers from the Swedish polyurethane foam industry: updated findings 1959-98
}

\author{
Z Mikoczy, H Welinder, H Tinnerberg, L Hagmar
}

Occup Environ Med 2004;61:432-437. doi: 10.1136/oem.2003.009712

\begin{abstract}
Aims: To assess whether cancer incidence and mortality in chronic obstructive lung diseases were increased in the Swedish polyurethane foam industry cohort, updated with 11 more years of follow up. Methods: The mortality and cancer incidence (1959-98) experienced by a cohort of 4175 male and female employees employed for at least one year in the period 1959-87 at one of nine Swedish polyurethane foaming plants were investigated. Comparisons were based on calendar year, sex, and fiveyear age group specific mortality and incidence rates for Sweden. Workplaces and job tasks were categorically assessed for exposure to tolvene diisocyanate (TDI) and methylene diphenyldiisocyanate (MDI) by occupational hygienists.

Results: Fewer cancer cases than expected were observed, but the lung cancer incidence was enhanced in women. Women with "apparent exposure" to TDI or MDI did not, however, have a higher lung cancer incidence than those with "no or low exposure". Moreover, a nested case referent study did not find that polyurethane dust exposure had been more prevalent among the female lung cancer cases than among referents. No increased mortality in chronic obstructive lung diseases was observed in the cohort.

Conclusions: Results support the findings from two other cohort studies of an increased lung cancer risk among female workers in the polyurethane foam manufacturing industry. Chance or confounding from smoking are not obvious explanations for the coherent findings. However, the study was not able to link isocyanate exposed employment with lung cancer risk.
\end{abstract}

See end of article for authors' affiliations

Correspondence to:

Prof. L Hagmar,

Department of

Occupational and

Environmental Medicine,

Lund University Hospital,

SE-221 85 Lund, Sweden;

lars.hagmar@ymed.lu.se

Accepted

2 November 2003

$\mathrm{T}$ oluene diisocyanate (TDI) and methylene diphenyldiisocyanate (MDI) are large volume industrial chemicals. The estimated yearly production in the early 1990s of TDI and MDI exceeded 2.5 million tonnes, and the production volumes have continued to grow since then.

TDI is usually produced as a mixture of the 2,4- and 2,6isomers. The main component of commercial MDI is $4,4^{\prime}$ MDI, but other isomers are also present in the product. The main use of these isocyanates is in the polyurethane foam manufacturing industry, but they are also used in the industrial production of coatings, adhesives, sealants, and various elastomers.

In a recent evaluation the International Agency for Research on Cancer considered that there is sufficient evidence that TDI is carcinogenic in experimental animals, and that there is limited evidence for a carcinogenic effect of MDI in animals. ${ }^{1}$ Inhalation of a mixture of monomeric and polymeric $4,4^{\prime}$-MDI increased the incidence of lung tumours in rats. ${ }^{2}$ Moreover, it has been reported that inhalation of freshly generated polyurethane dust generated lung tumours in rats. ${ }^{3}$

Results from three cohort studies of isocyanate exposed workers in the polyurethane foam manufacturing industry have been published. ${ }^{4-8}$ In the first report of the UK study, no notable increase of mortality in men was recorded for any specific cancer, while in women significant excesses of deaths were observed for cancers of the pancreas and lung. ${ }^{4}$ In internal analyses these risk enhancements could, however, not be related to isocyanates. Recently, the updated findings from the cohort, adding 10 more years of follow up, were reported. ${ }^{5}$ Furthermore, during these additional years of follow up, a significantly risk increase for female lung cancer was seen. For the total follow up period 35 lung cancer deaths were observed compared to 19.4 expected, giving a standardised mortality ratio (SMR) of 1.81. Internal analyses gave no indications that this risk enhancement could be attributed to isocyanate exposure.

No significantly increased cancer mortality was observed in a cohort study from the USA, but a non-significant risk excess in lung cancer mortality for women was noted. ${ }^{6}$

In the first report from the Swedish cohort study no overall excess in cancer incidence was observed, but risk increases for rectal cancer and non Hodgkin's lymphoma were found, when the first 10 years of follow up were ignored. ${ }^{7}$ A nested case-control study within the cohort did not support the tentative association between isocyanate exposure and these tumours. ${ }^{8}$ However, non-significant associations were shown for prostate cancer and colon cancer.

The main health concern with occupational exposure to TDI and MDI has been the risk for developing chronic airway disorders, especially bronchial asthma. ${ }^{9}$ It is not clearly established whether exposure to isocyanates in the polyurethane foam industry have affected the occurrence of chronic obstructive lung diseases to such an extent that it is reflected in increased mortality. In the recent update of the UK cohort there was in female, but not in male, workers a significant increasing trend of SMR for non-malignant diseases of the respiratory system with decade from hire. ${ }^{5}$ More detailed analyses gave, however, no support for the hypothesis that exposure to isocyanate was the causative factor. The results from the US cohort ${ }^{6}$ as well as the previous analysis of the Swedish cohort ${ }^{7}$ did not indicate any enhanced mortality in

Abbreviations: $D A B C O$, triethylenediamine; $E$, expected; $M D I$, methylene diphenyldiisocyanate; MOCA, methylenebis-o-chloroaniline; $\mathrm{O}$, observed; SIR, standardised incidence ratio; SMR, standardised mortality ratio; TDI, tolvene diisocyanate 
Main messages

- There was no overall risk increase for cancer among employees from the polyurethane foam industry.

- There was an increased lung cancer risk among female employees.

- The increased lung cancer risk among females could not be linked with exposure to TDI or MDI.

- There was no increased mortality in obstructive respiratory diseases among employees from the polyurethane foam industry.

non-malignant respiratory diseases, but this conclusion was based on relatively few observations.

The aim of the present study was to assess whether cancer incidence and mortality in chronic obstructive lung diseases were increased in the Swedish polyurethane foam industry cohort, updated with 11 more years of follow up.

\section{METHODS}

\section{The plants and chemical exposure}

Nine Swedish plants manufacturing polyurethane foam in which TDI or MDI had been used for producing flexible foam blocks or dead cast moulding materials were included in the study. ${ }^{7}$ The start of manufacture varied between 1958 and 1974. Table 1 gives the exposure periods for TDI and MDI for each plant.

The time weighted average concentrations of airborne TDI had normally been below $100 \mu \mathrm{g} / \mathrm{m}^{3}$ from 1965, when the first samples were taken, and below $20 \mu \mathrm{g} / \mathrm{m}^{3}$ at the end of the 1980s. The corresponding values for MDI were below $10 \mu \mathrm{g} / \mathrm{m}^{3}$. Much higher values (TDI up to $3 \mathrm{mg} / \mathrm{m}^{3}$; MDI up to $0.35 \mathrm{mg} / \mathrm{m}^{3}$ ) had, however, repeatedly been measured. Furthermore, there are reports of many accidental exposures to isocyanates due to spills and leakages. Thus, only five of the plants operated up to 1998. After 1990 TDI was used in only three of the plants. Due to its much lower vapour pressure, the use of MDI instead of TDI has led to lower emissions of isocyanates. The exposure levels have continuously been reduced over the years. In a large investigation performed by our department during the years 2000-01 of the airborne exposure to isocyanates in 13 plants in the Swedish polyurethane industry, the 113 monitored workers were exposed to total diisocyanates in the range 0.01-52 (median 4) $\mu \mathrm{g} / \mathrm{m}^{3}$ (CJ Sennbro, personal communication).

The workers could also have been exposed to other chemicals, for example, amine accelerators such as bis (2dimethylaminoethyl) ether, dimethylcyclohexylamine, dimethylethanolamine, ethanolamine, methylenebis-o-chloroaniline (MOCA), N-methylmorpholine, triethanolamine, triethylamine, and triethylenediamine (DABCO), and organic solvents such as petroleum, methylene chloride, methylethyl ketone, styrene, tetrachloroethylene, trichloroethane, toluene, and white spirit. These other chemicals have, however, rarely been monitored. A more detailed description has been given in a previous publication. ${ }^{7}$

\section{Cohort}

Name, date of birth, address, date of start and end of employment, and workplace or job task were obtained through the company records. Workers who had left employment or died before the year the company registers were complete were not included in the cohort. Seventy subjects whose personal identification code could not be retrieved and 686 subjects with missing data on start or end of employment

\section{Policy implications}

- The study results do not suggest that TDI and MDI are human carcinogens.

- The consistent, and up to now, unexplained risk increase for female lung cancer calls for future follow ups of employees from the polyurethane foam industry.

had to be excluded. The remaining 6426 workers had been employed for at least one day from 1958 to 1987 . The final cohort was restricted to those 4175 workers that had been employed for at least one year (table 1). Information on workplace or job task was available for all but 139 of these subjects.

The start of the observation period was defined as the first calendar year when both the foaming had started and the company records were considered to be complete and valid. This varied from 1958 to 1975 for the nine plants. The median calendar year of birth for the cohort members was 1951 (range 1896-1972), the median first year of exposure was 1978 (range 1958-86), and the median follow up period was 19.2 years (range 0.3-40). The Ethics Committee of Lund University approved the study.

\section{Exposure estimates and work tasks}

Each workplace and work task in the nine plants was categorically assessed for each calendar year up to 1987 by an experienced occupational hygienist for "no exposure", "low or intermittent exposure", or "apparent exposure" to TDI or MDI. The classification "apparent exposure" denotes direct exposure at the foaming or moulding processes or work in the same premises. The classification "low or intermittent exposure" denotes handling of cured materials in premises situated close by those used for foaming or moulding, or only intermittent exposures. Potential exposures after 1987 had to be disregarded because several of the plants had closed down since the previous analysis of the present cohort, which did not allow a valid retrospective exposure classification for the period after 1987.

All work periods could be classified with respect to exposure to TDI or MDI for 3988 of the 4175 cohort members. Of the exposure classified workers, 1394 had constantly experienced no, low, or intermittent exposure to TDI or MDI, while 1679 had constantly experienced apparent exposure. The remaining 915 subjects had varying exposure situations during their employment periods. The exposure-response

Table 1 TDI and MDI exposure periods for the nine polyurethane foam manufacturing plants and cohort sizes and person-years under observation for workers employed for at least one year

\begin{tabular}{lllrl}
\hline Plant & $\begin{array}{l}\text { TDI exposure } \\
\text { period }\end{array}$ & $\begin{array}{l}\text { MDI exposure } \\
\text { period }\end{array}$ & $\begin{array}{l}\text { Cohort } \\
\text { size }\end{array}$ & $\begin{array}{l}\text { Person-years } \\
\text { under } \\
\text { observation }\end{array}$ \\
\hline A & $1959-73$ & $1968-98$ & 262 & 6560 \\
B & $1969-95$ & $1969-95$ & 71 & 1681 \\
C & $1973-90$ & $1982-90$ & 146 & 2712 \\
D & $1958-98$ & Not known & 398 & 7872 \\
E & $1962-98$ & $1972-98$ & 404 & 9020 \\
F & $1972-92$ & $1972-92$ & 379 & 6632 \\
G & $1981-83$ & $1970-98$ & 955 & 17414 \\
H & $1958-74$ & $1964-98$ & 1038 & 20482 \\
I & $1977-87$ & $1966-98$ & 522 & 10650 \\
Total & & & 4175 & 83023 \\
\hline
\end{tabular}



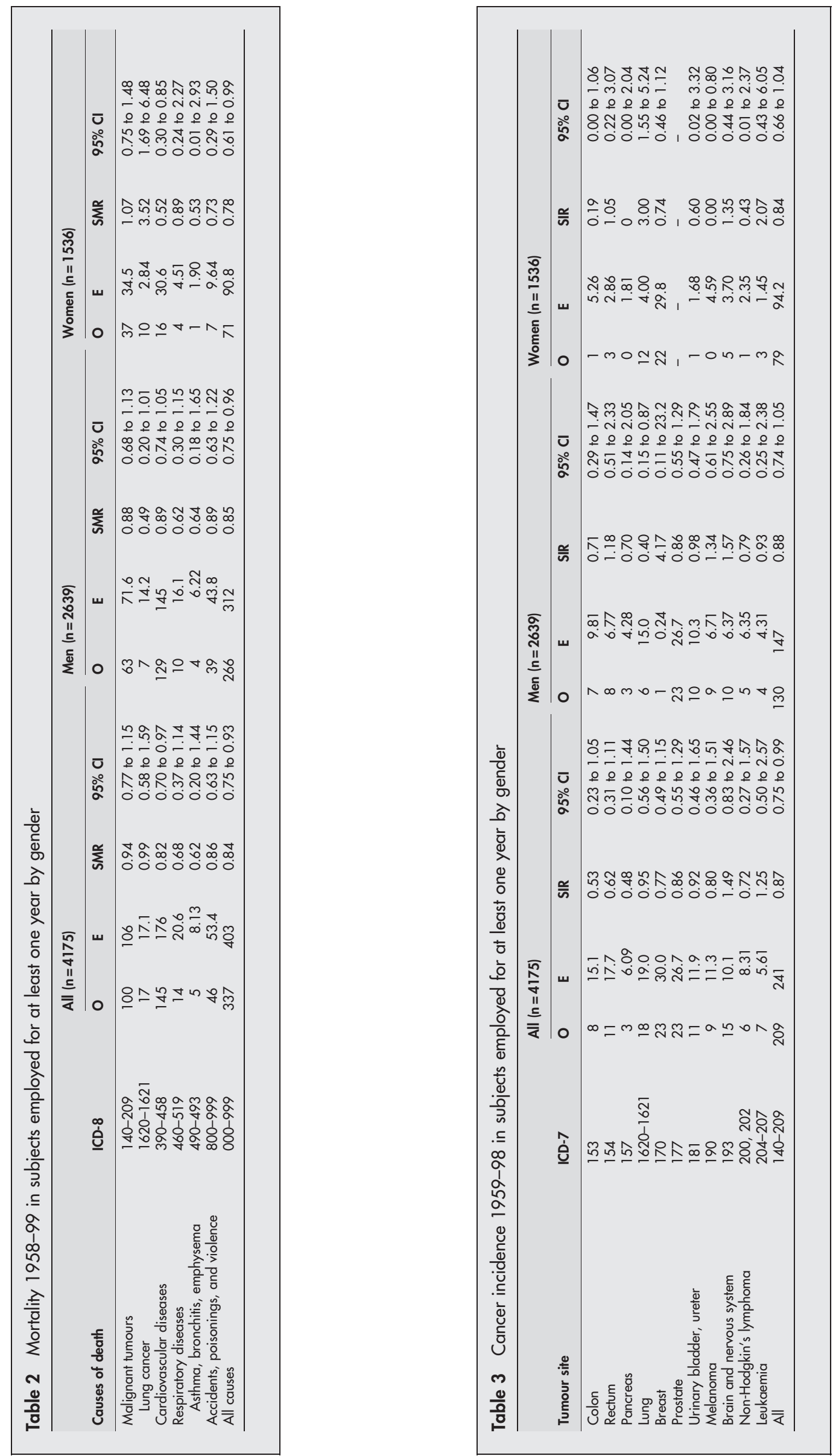

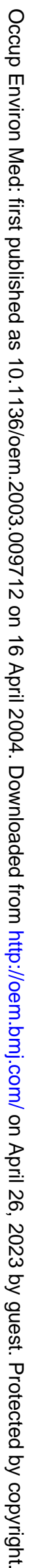


Table 4 Cancer incidence 1959-98 in 4175 subjects employed for at least one year and after at least 20 years since first exposure

\begin{tabular}{llcccc}
\hline Tumour site & ICD-7 & O & E & SIR & $\mathbf{9 5 \% ~ C l ~}$ \\
\hline Colon & 153 & 4 & 5.58 & 0.72 & 0.19 to 1.84 \\
Rectum & 154 & 1 & 3.48 & 0.29 & 0.01 to 1.60 \\
Lung & $1620-1621$ & 8 & 6.74 & 1.19 & 0.51 to 2.34 \\
Breast & 170 & 9 & 9.69 & 0.93 & 0.42 to 1.76 \\
Prostate & 177 & 12 & 11.4 & 1.05 & 0.54 to 1.84 \\
Urinary bladder, ureter & 181 & 4 & 4.39 & 0.91 & 0.25 to 2.33 \\
Melanoma & 190 & 1 & 3.04 & 0.33 & 0.01 to 1.83 \\
Brain and nervous system & 193 & 6 & 2.45 & 2.45 & 0.90 to 5.33 \\
Non-Hodgkin's lymphoma & 200,202 & 1 & 2.89 & 0.35 & 0.01 to 1.93 \\
Leukaemia & $204-207$ & 2 & 1.66 & 1.20 & 0.15 to 4.35 \\
All & $140-209$ & 80 & 80.5 & 0.99 & 0.78 to 1.23 \\
\hline
\end{tabular}

calculations were restricted to the 3073 subjects with stable exposure classifications over time.

There was a gender difference with respect to work tasks in the plants. Twenty six per cent of the female workers had been involved in the finishing treatment of the foamed products compared with $12 \%$ of the male workers. These work tasks, comprising grinding, punching, sawing, and cutting, may have resulted in polyurethane dust exposure. Unfortunately, no dust measurements had been performed in the plants.

\section{Information on causes of death and tumours and risk estimates}

Information on cause of death for the period 1959-98, coded according to the International Classification of Diseases (ICD), 8th revision, was obtained from Statistics Sweden. Information on malignant tumours for the period 1959-98 coded according to the ICD, 7th revision, was obtained from the Swedish Cancer Registry. Information on vital status and date of emigration was obtained from the Swedish population registry. Three hundred and thirty seven of the 4175 workers were deceased at 31 December 1998, 123 had emigrated, and the rest were still alive and living in Sweden. None was lost to follow up.

Expected mortality and cancer incidence for the same period was calculated by means of the SYDCAP cohort program using cause, calendar year, sex, and five-year age group specific rates. These rates were calculated from incidence rates for specific causes of death and malignant tumours and population counts, obtained from Statistics Sweden and the Swedish Cancer Registry. Date of death, emigration, or a person's 80th birthday were used as individual endpoints, whichever occurred first. Altogether the cohort contributed 83023 person-years under risk (table 1). The $95 \%$ confidence intervals (95\% CIs) for cause specific standardised mortality and incidence ratios (SMRs and SIRs) were calculated by treating the observed number as a Poisson variable, or as a normal variable if the observed value was greater than 15 . The term significant indicates that the $95 \%$ CI does not include 1.00 .

\section{Nested case-referent study}

For each of the 12 female lung cancer cases, three referent women from the cohort without cancer were randomly selected but matched for calendar year of birth. Two occupational hygienist (HT, HW) used available information for each subject about plant and workplace or job task, to crudely classify the likelihood that the subjects had been substantially exposed to polyurethane dust. Due to lack of exposure data, no classification could be performed for one of the cases. Odds ratios and 95\% CIs were calculated using conditional logistic regression.

\section{RESULTS}

A total of 337 workers had died during the observation period, which was significantly fewer than expected (SMR $0.84,95 \%$ CI 0.75 to 0.93 , table 2 ). No increased mortality was observed for either malignant tumours or for chronic obstructive lung diseases. An increased mortality in lung cancer was observed, however, only in women (SMR 3.52, $95 \%$ CI 1.69 to 6.48 ).

Two hundred and nine incident cancer cases were observed, which was fewer than expected (SIR 0.87, 95\% CI 0.75 to 0.99 , table 3$)$. The lung cancer incidence was enhanced in women (SIR 3.00, 95\% CI 1.55 to 5.24), but not in men. Seven of the 12 women with incident lung cancer had also been registered as deceased from this disease, while two had died of intercurrent diseases and three were still alive.

A numerical increase in malignant neoplasms in the brain and nervous system was observed (SIR $1.49,95 \%$ CI 0.83 to 2.46), which did not seem to differ between men and women. The incidences of rectal cancer, non-Hodgkin's lymphoma, and pancreatic cancer were not increased.

The overall cancer incidence was not increased, even when lagging the first 20 years since start of exposure, but the SIR

Table 5 Exposure to TDI or MDI and cancer incidence in polyurethane foam industry workers with stable exposure classification over their employment period

\begin{tabular}{|c|c|c|c|c|c|c|c|c|c|c|c|c|}
\hline \multirow[b]{3}{*}{ Tumour } & \multirow{2}{*}{\multicolumn{4}{|c|}{$\begin{array}{l}\text { None or constantly low exposure } \\
(n=1394)^{*}\end{array}$}} & \multicolumn{8}{|c|}{ Constantly apparent exposure $(n=1679) \dagger$} \\
\hline & & & & & \multicolumn{4}{|c|}{$>5$ years employment } & \multicolumn{4}{|c|}{ All employment periods } \\
\hline & 0 & $\mathbf{E}$ & SIR & $95 \% \mathrm{Cl}$ & 0 & E & SIR & $95 \% \mathrm{Cl}$ & 0 & $\mathbf{E}$ & SIR & $95 \% \mathrm{Cl}$ \\
\hline Lung cancer (women) & 6 & 1.50 & 4.00 & 1.47 to 8.71 & 3 & 0.63 & 4.76 & 0.98 to 13.9 & 5 & 1.53 & 3.27 & 1.06 to 7.64 \\
\hline Brain cancer (men and women) & 6 & 3.68 & 1.63 & 0.60 to 3.55 & 1 & 1.32 & 0.76 & 0.02 to 4.22 & 5 & 3.90 & 1.28 & 0.42 to 3.00 \\
\hline All (men and women) & 80 & 92.2 & 0.87 & 0.69 to 1.08 & 27 & 36.9 & 0.73 & 0.48 to 1.06 & 82 & 93.4 & 0.88 & 0.70 to 1.09 \\
\hline
\end{tabular}


Table 6 Nested case referent study of the association between exposure to polyurethane dust and female lung cancer incidence

\section{Likelihood for polyurethane} dust exposure Cases Referents

\section{Low} High

$7 \quad 20$
14

Odds ratio 0.8 (95\% Cl 0.2 to 2.9 )

for malignant neoplasms in the brain and nervous system became somewhat higher $(2.45,95 \%$ CI 0.90 to 5.33 , table 4$)$.

There were no indications of any exposure-response associations between level or length of TDI or MDI exposure and total cancer incidence, or brain cancer or female lung cancer (table 5).

The results from the nested case referent study did not indicate that polyurethane dust exposure had been more prevalent among the female lung cancer cases than among the referents (table 6).

\section{DISCUSSION}

The main result of the present study was the increased lung cancer incidence in female workers in the polyurethane foam manufacturing industry, which is noteworthy because this has also been observed in the UK and the US cohorts. A significant lung cancer mortality excess (SMR 1.81, 95\% CI 1.26 to 2.51 ) was seen in female workers in the UK cohort, ${ }^{5}$ and in the US cohort ${ }^{6}$ there was non-significantly increased lung cancer mortality among the female workers (SMR 1.73, $95 \%$ CI 0.75 to 3.41 ).

The reason for this consistent pattern of increased female lung cancer risk is not obvious. In both the UK study ${ }^{5}$ and the present study there were attempts to assess exposureresponse associations with respect to estimated exposure to TDI or MDI, but no such patterns were found. In the US study there were no attempts to perform dose-response analyses. ${ }^{6}$ The lack of dose-response associations in the UK study and the present study might speak against a causal role for isocyanate exposure. On the other hand, the exposure levels for TDI and MDI have varied considerably both between different workplaces in the plants and within them. The information derived from the company records on the workplace and job tasks for the subject in the cohort has not always been specific, at least not in the Swedish cohort. Taken together, this has probably caused a non-differential misclassification of exposure, which will decrease the possibility to associate a risk excess with specific exposures. Moreover, the present exposure-response analysis, based on only 11 lung cancer cases, has a low statistical power to detect more than very high risk increases associated with high exposure levels.

The manufacture of polyurethane foam involves exposure not only to isocyanates but also to a number of other chemicals, which unfortunately rarely have been monitored, such as expanding agents, lubricants, accelerators, and organic solvents. The use of these compounds has varied between the plants involved in the present study, and the exposure to isocyanates has thus been the only common chemical characteristic of the plants. In the first report of the UK study an attempt was made to assess the risk for female lung cancer associated with other types of chemicals by a nested case referent analysis. ${ }^{1}$ No obvious pattern was seen, but the statistical power was low due to the few cases and referents.

A larger fraction of female workers than of male workers had had work tasks that potentially could have lead to airborne exposure to polyurethane dust. The nested case- referent analysis in the present study did, however, not support exposure to polyurethane dust as a risk factor for female lung cancer. It should, however, be emphasised that very few dust measurements had been performed at the plants and the exposure could therefore be only crudely assessed. This, together with the small size of the study limited the possibility to evaluate more than very strong associations between polyurethane dust and lung cancer.

Unfortunately no information on smoking habits was available for the UK, ${ }^{5}$ the USA, ${ }^{6}$ or the present cohort. There is, however, circumstantial evidences that makes smoking a less likely explanation for the excess risk for female lung cancer. First, no increase in male lung cancer was observed in any of the three cohorts (UK cohort: SMR 1.07; US cohort: SMR 0.79; present study: SIR 0.40). Thus, if smoking has caused the increased risk for lung cancer among the female workers, we have to assume very different smoking habits between male and female workers. Second, smoking is a risk factor for cardiovascular mortality, but the corresponding SMRs for females were not increased in any of the three cohorts (UK cohort: 1.09; US cohort: 0.73; present study: 0.52 ). The age related increase in cardiovascular mortality occurs later in women than in men due to the protective effect of endogenous oestrogens. The low SMR for cardiovascular diseases for women in the present cohort was not the result of a still too young cohort as the SMR for the age category 70 years and above was 0.55 .

No increased mortality in respiratory diseases, or more specifically in chronic obstructive lung diseases, was observed in the present study or in the US study. ${ }^{6}$ In the UK study female workers had a significant trend of SMRs for nonmalignant respiratory diseases increasing with decade from hire. ${ }^{5}$ However, no association with isocyanates exposed employment was observed. Thus, there is no convincing evidence that exposure to isocyanates in the polyurethane foam manufacturing industry has resulted in increased mortality in obstructive lung diseases.

To conclude, the results from the present study support the findings from two other cohort studies of an increased lung cancer risk among female workers in the polyurethane foam manufacturing industry. However, no doseresponse associations with occupational exposure to TDI or MDI have been observed. A caveat is, however, the inherent limitations of retrospective exposure assessments. A chance finding or confounding from smoking are not obvious explanations for the risk excesses. An occupational risk factor cannot yet be excluded and a prolonged follow up of all three cohorts with more in depth exposure analyses could contribute to a better understanding of the observations.

\section{ACKNOWLEDGEMENTS}

The work was financially supported by grants from AFA-insurances, the Medical Faculty, Lund University, Region Skåne, and Lund University Hospital.

\section{Authors' affiliations}

Z Mikoczy, H Welinder, H Tinnerberg, L Hagmar, Department of Occupational and Environmental Medicine, Lund University Hospital, Lund, Sweden

\section{REFERENCES}

1 International Agency for Research on Cancer. IARC monographs on the evaluation of the carcinogenic risk of chemicals to humans. Re-evaluation of some organic chemicals. Vol 71. Lyon: IARC, 1999.

2 Reuzel PGJ, Arts JHE, Lomax LG, et al. Chronic inhalation toxicity and carcinogenicity study of respirable polymeric methylene diphenyl diisocyanate (polymeric MDI) aerosols in rats. Fundam Appl Toxicol 1994;22:195-210.

3 Stemmer KL, Bingham E, Barkley W. Pulmonary response to polyurethane dust. Environ Health Perspect 1975;11:109-13. 
4 Sorahan T, Pope D. Mortality and cancer morbidity of production workers in the United Kingdom flexible polyurethane foam industry. Br J Ind Med 1993;50:528-36.

5 Sorahan T, Nichols L. Mortality and cancer morbidity of production workers in the UK flexible polyurethane foam industry: updated findings, 1958-98. Occup Environ Med 2002;59:751-8.

6 Schnorr TM, Steenland K, Egeland GM, et al. Mortality of workers exposed to toluene diisocyanate in the polyurethane foam industry. Occup Environ Med 1996;53:703-7.
7 Hagmar L, Welinder H, Mikoczy Z. Cancer incidence and mortality in workers from the polyurethane foam manufacturing industry. $\mathrm{Br} J$ Ind Med 1993;50:537-43.

8 Hagmar L, Strömberg $\mathrm{U}$, Welinder $\mathrm{H}$, et al. Incidence of cancer and exposure to tolvene diisocyanate and methylene diphenyldiisocyanate: a cohort based case referent study in the polyurethane foam manufacturing industry. $\mathrm{Br} J$ Ind Med 1993;50:1003-7.

9 Baur X, Marek W, Ammon J, et al. Respiratory and other hazards of isocyanates. Int Arch Occup Environ Health 1994;66:141-52.

\section{$\mathrm{ECHO}$}

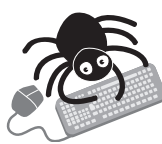

Please visit the Occupational and Environmental Medicine website [www. occenvmed. com] for a link to the full text of this article.

\section{Asthma in domestic cleaners}

ecent studies have suggested that cleaners, especially domestic cleaners, may have an increased risk of asthma. A study in Barcelona, Spain has confirmed this increase in risk.

In a population thought to have a high number of domestic cleaners a previously validated questionnaire was sent to 5120 women aged 30-65 and with less than eight years' education. Telephone follow up was employed as necessary. Satisfactory replies were received from 4521 women of whom half (2259) had worked as cleaners at some time and 593 (13\%) were currently working as domestic cleaners. Most of the women $(74 \%)$ had never smoked. Relative to women who had never worked in cleaning, women who had ever worked in cleaning had significant increases in wheezing with breathlessness (odds ratio (OR) 1.53), ever asthma (1.44), current asthma (1.73), chronic bronchitis (1.52), and ever rhinitis (1.2). The risks were increased for current cleaners but the increases were significant only for wheezing with breathlessness, current asthma, and chronic bronchitis in domestic, but not non-domestic, cleaners. The highest risks were in former domestic cleaners (OR for current asthma 2.09) but few former cleaners (1.3\%) claimed to have quit the job because of respiratory symptoms. In general neither current nor previous non-domestic cleaning was significantly associated with asthma. Women who worked simultaneously as domestic and non-domestic cleaners had a higher risk of asthma (OR 1.79) than women who did only domestic cleaning. Among current non-domestic cleaners the asthma risk was increased for hospital or health centre cleaners but not for office, hotel, residence, laboratory, or kitchen cleaners. About a quarter of the asthma cases in this study population could be attributed to domestic cleaning work.

Domestic cleaning could have an important effect on public health, especially if the increased risks were found to apply to household members doing cleaning as well as paid cleaners. The precise causes of the increased asthma risk in domestic cleaners are unknown.

A Thorax 2003;58:950-954. 